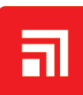 CHITKARA \\ Journal of Nuclear Physics, Material Sciences, Radiation and Applications

\section{Study of the Erosion of Copper by Hot Plasma}

\author{
R. S. Monzamodeth and B. Campillo* \\ Chemistry Faculty, National Autonomous University of Mexico (UNAM), PO Box 04510 Mexico City, Mexico
}

*Email: bci@icf.unam.mx

\section{ARTICLE INFORMATION}

Received: October 10, 2019

Accepted: February 4, 2020

Published online: February 28, 2020

\section{Keywords:}

Copper, Hot plasma, Erosion, Hardness, Plasma/Wall interactions, Hydrophobic properties

\begin{abstract}
An exhaustive study of the erosion process of a copper cathode exposed to a hot plasma column of $2 \mathrm{~kJ}$ of energy $(\mathrm{T} \approx 0.5-2.0 \mathrm{keV})$ and high electron density $\left(\mathrm{n} \approx 1019-1022 \mathrm{~cm}^{3}\right)$ was made, as well as, the radiation field of charged and neutral particles. The characterization of the cumulative damage generated by the plasma/cathode interaction was made by the use of metallographic techniques, scanning electron microscopy (SEM) and by the analysis of mechanical properties. Damage accumulation produced by the impacts of deuterium plasma discharge created in the copper electrode a deep cavity similar to a crater, modifying the morphology of the surface and below it. The microhardness Vickers test was carried out making indentations from the final part of the cavity to cover $1 \mathrm{~cm}$ with indentations every $200 \mu \mathrm{m}$. Different areas of hardening were observed, the profile suggests a hardening/recovery front and simultaneous recrystallization in the sample, phenomenon associated with the heating/cooling cycles to which the copper cathode is subjected. Images were captured by SEM at different distances from the center of the surface. The region that showed involvement at the macro level corresponds to $2 / 3$ of the radius of the sample from the center to the outside. These phenomena studied are important to understand the nature of the plasma/wall interaction in any fusion device.
\end{abstract}

\section{Introduction}

The dense plasma focus (DPF) or plasma focus (PF) is a device by which it is possible to produce a plasma column of high density and temperature. The design is relatively simple compared to other nuclear fusion concepts. This type of device consists essentially of coaxial plasma cannon, whose electrodes are isolated in the chamber, being the internal electrode the anode and the cathode the external [1]. After charging a capacitor bank, the energy is transmitted to the device by a gas switch in an order time of 20-50ns. As a result, the discharge breaks on the insulator surface, ionizing the gas that fills the chamber at a pressure of a few Torr. The resulting radial current sheet is propelled to the mouth of the cannon by the magnetic field generated by it. Upon reaching the opposite end of the internal electrode, this sheet focuses on a dense plasma column, confined by the magnetic field of the current, which represents an interesting source of radiation. If deuterium is used as a charge gas, fusion reactions occur through the ${ }^{2} \mathrm{H}+{ }^{2} \mathrm{H}$ $\rightarrow{ }^{3} \mathrm{He}(0.8 \mathrm{MeV})+n(4.5 \mathrm{MeV}) \mathrm{y}^{2} \mathrm{H}+{ }^{2} \mathrm{H} \rightarrow{ }^{3} \mathrm{He}(1.0$ $\mathrm{MeV})+{ }^{1} \mathrm{H}(3.0 \mathrm{MeV})$ channels. While the plasma emits soft X-rays due to thermal Bremsstrahlung, instabilities in the plasma produce intense electric fields, resulting in the generation of ions and electrons that are collimated axially; the first in the opposite direction to the barrel and the second on the anode, emitting a hard X-ray pulse, and evaporating electrode material. This type of device is not an appropriate candidate to design a nuclear reactor, however it is a promising source of radiation, therefore its study is of great interest both for the physics involved and for its necessary applications [1-3]. X-rays in a plasma focus can have different origins. There are soft $\mathrm{X}$-rays, which arise from thermal Bremsstrahlung in the plasma and can be observed inside the chamber by pin diodes, recorded on film with vacuum chambers, covering the holes with filters or combining both techniques using the diodes instead of the film. Because there are generally impurities in the plasma, linear spectra can also be observed. Planting impurities, such as argon or xenon, is a common practice. For example, many authors have studied the efficiency of K-series argon line radiation in an argon-operated device [4]. However, the most energetic radiation comes from an electron beam well in the region of tens of $\mathrm{keV}$, generated within the plasma column when it collides with the internal electrode [5]. Such radiation can be observed outside the chamber and used for radiographic purposes. In a previous work [6] radiographs are showed obtained using a solid internal electrode, which 
after several shots showed a very thin hole, $3 \mathrm{~mm}$ wide and $6 \mathrm{~mm}$ deep, this was perforated by the electron beam, which means that it is very well focused. The key to high contrast radiography is to have a bright point source. Copper have an excellent electrical and thermal conductivity, therefore, its use is common in applications where high electrical or thermal conductivities are required. An important consideration related to the electrical and thermal properties of copper is the fact that even small amounts of impurities negatively affect its electrical conductivity. This is because any irregularity within the space network of a metal increases the dispersion of electrons and induces a reduction in its mean free path. Consequently, the purity of copper for use in cables and other forms used in the transport of electricity must be carefully controlled [8]. Oxygen-free or high-conductivity copper generally refers to a group of high conductivity copper alloys forged that has been electrolytically refined to reduce the oxygen level to $0.001 \%$ or below this concentration.

The interaction of the plasma wall refers to phenomena that occur as a result of plasma contact with a solid body, not only on the contact surface, but also within the plasma and solid [9-12]. When the plasma is in contact with a solid (which generally occurs in artificially generated plasmas), the solid acts as a sink for the plasma. The ions and electrons precipitate in the solid and recombine, emerging as neutral atoms that reionize within the plasma, usually by collision with electrons. The solid acts as a sink for the plasma, at the same time feeding with neutrals atoms the source that generates new plasma in its vicinity. This phenomenon is known as plasma recycling. The rationale for this phenomenon is explained below.

In plasma, electrons have great mobility compared to ions due to the difference in mass between them. This causes electrons to diffuse rapidly; but an electric polarization field is also created by the separation of the charges that in the absence of another force will return the flow of electrons, restoring the quasi-neutrality of the plasma. Therefore, electronic diffusion is limited by ionic diffusion. This process is known as ambipolar diffusion. Due to this mobility difference, electrons will be the first species to reach a surface exposed to plasma. This causes the appearance of an electrical potential gradient in the plasma in contact with the surface, a gradient that is quickly attenuated by the effect of shielding charges (distances of Debye lengths order).

The region in which the potential falls from its value in the plasma to the "floating potential" of the surface is called a sheath, or Debye's sheath, to differentiate it from the magnetic sheath. In this work we use the same small $4.6 \mathrm{~kJ}$ dense plasma focus device to test how contrast in radiographs can be improved. Also using TLD-100 dosimeters we determine the average dose received by the sample. In order to improve the punctual nature of the source we use Tungsten needles implanted at the tip of the electrode and study the effects of needle deterioration on the central (copper) electrode. The internal electrode made of oxygen-free copper is $40 \mathrm{~mm}$ long with a diameter of $50 \mathrm{~mm}$ [1]. Two different Tungsten needles were tested; one $1 \mathrm{~mm}$ wide and $6 \mathrm{~mm}$ long and another $2 \mathrm{~mm}$ wide and $6 \mathrm{~mm}$ long. These bright spots were generated at the tip of the needle, which were deteriorated by plasma and electron beam. The destruction of the needles affects the plasma and the electrodes so it was necessary to study them to observe the effects on the electrode $[6,7]$.

\section{Experimental Setup}

This work was performed using the FN-II small plasma focus device, operated at the ICN-UNAM [1]. It is a $4.8 \mathrm{~kJ}$ device at $38 \mathrm{kV}$, with an oxygen-free copper anode, $40 \mathrm{~mm}$ long, with a $50 \mathrm{~mm}$ diameter. The co-axial cathode is formed by ten copper rods arranged in a squirrel cage configuration with a radius of $50 \mathrm{~mm}$. The insulators are an annular Pyrex tube located at the base of the anode, matching its diameter. The energy storage is provided by four $1.863 \mu \mathrm{F}$ capacitors in parallel, and the discharge is triggered by a simple mushroom electrode spark gap. Throughout the present work the plasma focus was operated in its neutron optimized regime, corresponding to a 2.75 Torr deuterium gas pressure. In this regime a peak focus current of $350 \mathrm{kA}$ and an average neutron yield of $\sim 3 \times 10^{8}$ per shot are obtained. The evolution of the current derivative is obtained with a Rogowski coil. Two silver foil activation counters, one on axis and the other at $90^{\circ}$, were used to measure the neutron yield, Fig. 1.

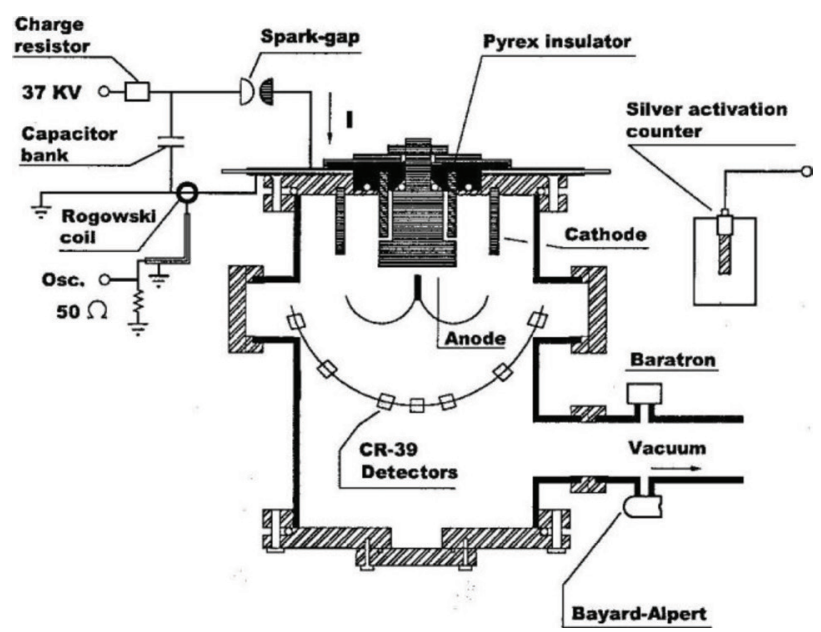

Figure 1: Diagram of the Fuego Nuevo II PF device, also shown the diagnostic equipment used. 
The anode electrode was machined from oxygen free copper with a Tungsten core with 1 to $2 \mathrm{~mm}$ in diameter (Tungsten needle) located at the center of the copper electrode. The length of the needles was monitored by a fivehole chamber in which the $100 \mu \mathrm{m}$ holes are covered with 5 , $10,15,20$ and $25 \mu \mathrm{m}$ aluminum filters [6]. The bright spot on the tip of the needle can be clearly seen in the image in Fig. 2, while erosion of the 1 and $2 \mathrm{~mm}$ needles depending on the number of plasma discharge is shown in Fig. 3 [7].

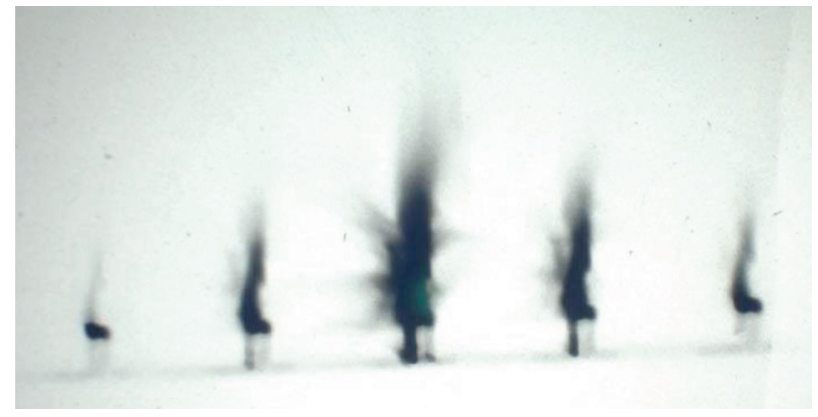

Figure 2: (Color online) Multipinhole camera images of the plasma column with a needle, filtered by $25,15,5,10$, and $20 \mu \mathrm{m}$ of aluminum foil.

The hardness test measures the surface resistance of a material to penetration. Hardness is defined as the greater or lesser resistance that opposes one body to be scratched or penetrated by another. The variables to take into account when performing a hardness test on the materials are the applied load, the geometry of the indenter, the size of the imprint and the time lap of load [18]. In the Vickers hardness test the indenter is shaped like a regular squarebased pyramid in which the angle formed by two opposite faces is $136^{\circ}$. The specifications that the indenter must cover are very strict. The ASTM standards require that the angle between the faces does not differ from $136^{\circ}$ by more than 30 ". Also, the standard demands the four faces meet at one point and that any defect of the diamond is less than $5 \%$ of the diagonal of the impression that it produces [18].

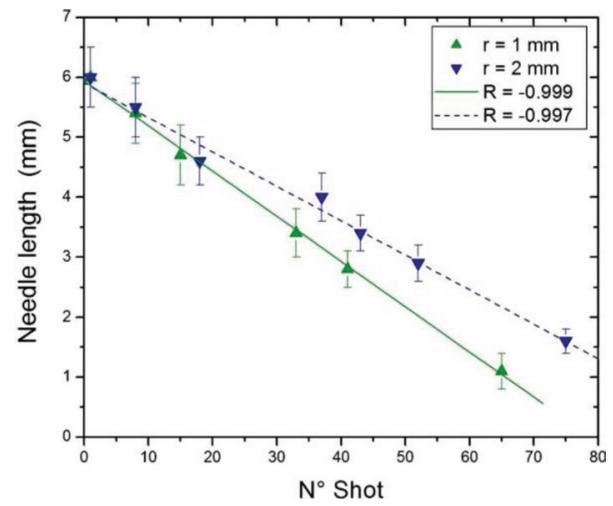

Figure 3: (Color online) Needles erosion as a function of the shots numbers.
For the Vickers micro-hardness test, the longitudinal central face along to the Tungsten core was used. The microhardness test was carried out in a LECO LV810AT microdurometer using a load of $100 \mathrm{gf}$ (which was determined by means of a calibration curve) and a time of 20 s of load. On this longitudinal central face, half of said face, microhardness was measured to see the effect of heating/cooling cycle produced by plasma shooting in the copper electrode. Several measurements were made on the longitudinal surface to determine the microhardness distribution in this copper electrode surface. Several measurements were made on the longitudinal surface to determine the inner microhardness distribution in copper electrode.

On the upper surface, the formation of a morphology layer different from that of the copper electrode was observed; this was analyzed measuring the wetting angle by the static drop method and SEM. To determine the wettability behavior of the sample, on both sides after plasma treatment, the sessile drop technique was used to measure the contact angle (CA) with distilled water (drop volume: $5 \mu \mathrm{L}$ ). Using a USB digital microscope (Model Px-537) the contact angle was determined in three areas of the surface with deposited film. The contact angle was calculated by analyzing the images of the drops with the Image $\mathrm{J}^{\bullet}$ program.

In order to observe the change in the surface morphology of the electrode due to the wear of the Tungsten needle by the plasma, the samples were analyzed by Scanning Electron Microscopy (SEM). Images were taken at different magnifications and at different points to characterize the Copper/Tungsten electrode surface. The equipment used was a SEM JEOL JSM-5900LV located at USAII, UNAM. The damage generated by the plasma/surface interaction has been studied by other researchers [13-17].

\section{Results}

Vickers microhardness test was performed using a work load of 100gf. Fig. 4 shows the area that was measured to determine the microhardness profile across the longitudinal central face of the copper electrode after interacting with the plasma; this shows the changes in hardness as a function of the depth (A) and radial position (B) over copper electrode. Figure $4 \mathrm{a}$ and $4 \mathrm{~b}$ show the $2 \mathrm{D}$ and $3 \mathrm{D}$ microhardness corresponding to the "mesh of indentations" corresponding to the axis "A" and "B". The axis "A" has a size of $1.5 \mathrm{~cm}$ and the "B" $1 \mathrm{~cm}$. Therefore, the analyzed area was $1.5 \mathrm{~cm}^{2}$. Each ideation is separated by $1 \mathrm{~mm}$ of distance, finally obtaining a total of 168 indentations. This spatial distribution of the microhardness values is associated with the high energy impact over the electrode surface during the plasma discharges and the heating/cooling 
cycle imposed. This cycle is imposed in nanoseconds and modifies the mechanical properties. The electron high energy impact produce hardening by plastic deformation still undersurface; the top surface is melted forming a very thin film. Then, the plastically deformed copper under the surface is annealed due to heating process until its cooling by the copper heat conductivity. This can be observed in Fig. 4a, there is a hardened region ( $101 \mathrm{HV} 0.3$ ) at coordinates $(0.1,0.1)$, a is led by a region with lower hardness between 78 to $91 \mathrm{HV} 0.3$ and finally there is a hardness increase (104 HV0.3) at reach the coordinate $(0.85,1.25)$. Fig. $4 \mathrm{~b}$ shows the 3D microhardness mapping across the analyzed area, the area colored in red correspond with the upper values meanwhile the blue colored areas correspond with the lower microhardness values.

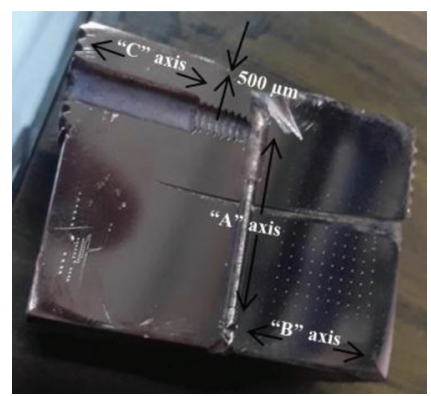

Figure 4: Microhardness evaluation zone.

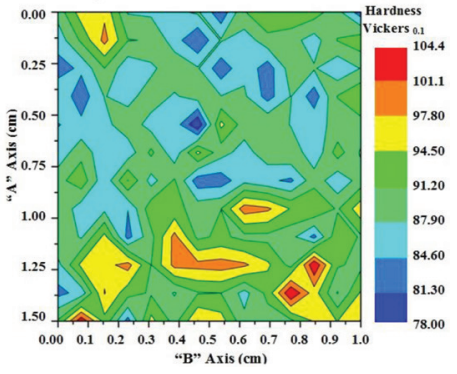

Figure 4a: 2D microhardness mapping.

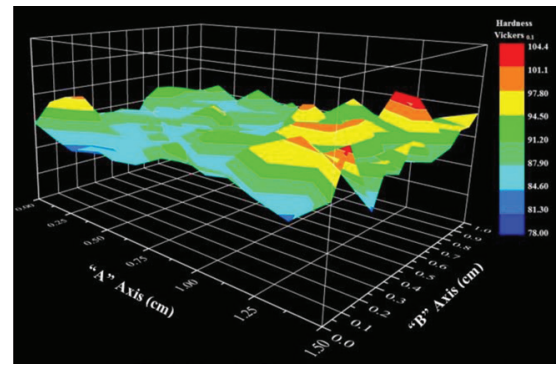

Figure 4b: 3D microhardness mapping.

A microhardness test was also carried out very close to the edge, right in the area of greatest interaction with plasma. Fig. 5 shows the microhardness results on the " $\mathrm{C}$ ", which has a length of $1 \mathrm{~cm}$ and is at a distance of $500 \mu \mathrm{m}$ from the edge. The indentations were also separated at a distance of $1 \mathrm{~mm}$ between them, obtaining 16 measurements along the "C" axis. The green point $\bullet$ represents the beginning of the analysis located on the left end of the "C" axis, while the red point represents the end of the test, located on the right end of the same axis. In this image can observe that the microhardness increases from the border ( $96 \mathrm{HV} 0.3$ ) to centerline of Tungsten core in the copper electrode (135 HV0.3).

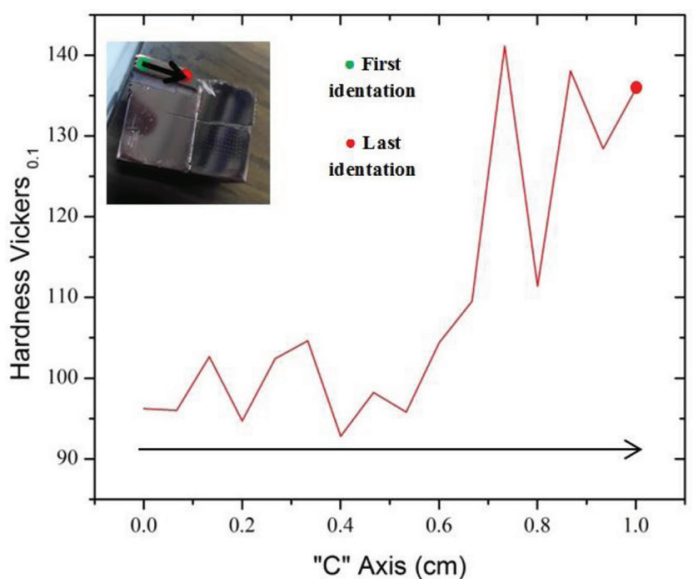

Figure 5: Results of the hardness test on the " $C$ " axis.

Using a digital microscope (Model Px-537) the sessile drop technique was used to measure the contact angle (CA) with a $5 \mu \mathrm{L}$ distilled water drop. The CA was determined in three different regions of the upper surface of the electrode (the part that was covered by the molten Tungsten). ImageJ software was used for the analysis of the 3 drops. The obtained values $\left(>90^{\circ}\right)$, see Fig. 6, confirm that the surface is hydrophobic character, this behaviour is produced by both the surface morphology and the surface chemical composition. The maximum CA value and the maximum hydrophobicity is located where the deposited film is thicker, near the copper electrode center around the Tungsten core.

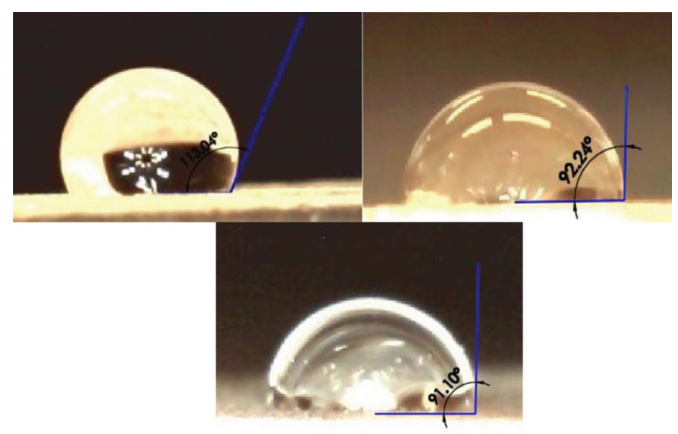

Figure 6: Sessile drops of $5 \mu \mathrm{L}$ in volume of distilled water over top copper electrode surface. The Tungsten film over copper electrode has a hidrophobic character. 
Scanning Electron Microscope (SEM) analysis (Fig. 7-9) showed a change in the morphology across the sample surface, from the central region to the surface near to the border. Also in X-ray energy dispersive spectroscopy (EDS) elementary mapping, it can be observed the formation of a layer of Tungsten apparently molten over the copper electrode. This film looks thicker near the centerpiece region and thinner near the electrode border. This film was formed due to the rapid heating experienced by the sample when was subjected to the hot plasma shots that were made in the FN-II small PF device. The plasma generated in each shot is hot plasma, although only about 70 shots (plasma discharge) were taken.

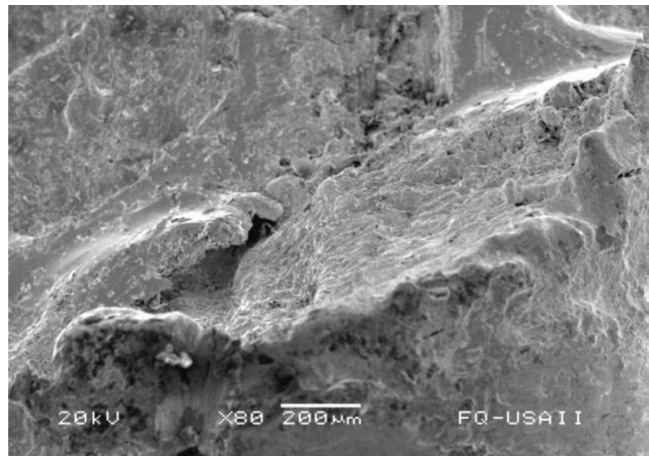

Figure 7: Secondary electron image (SEI), general appearance of the Tungsten needle eroded surface and melted film over copper electrode. A layer of approximately 100 microns partially melted, cracked in several regions.
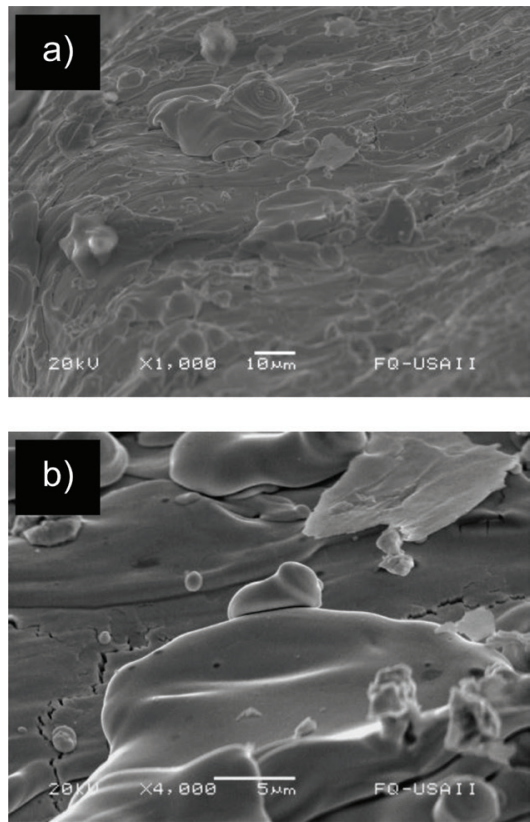

Figure 8: a) Detail of the copper electrode surface covered by molten Tungsten. The surface morphologies show the form of drops with varied sizes from 1 micron to about 30 microns. These morphologies must be generated by the high energy beam impact process. b) In the right picture it can be observed partially cast surface with cracking and solidified molten droplets with less than 1 micron.
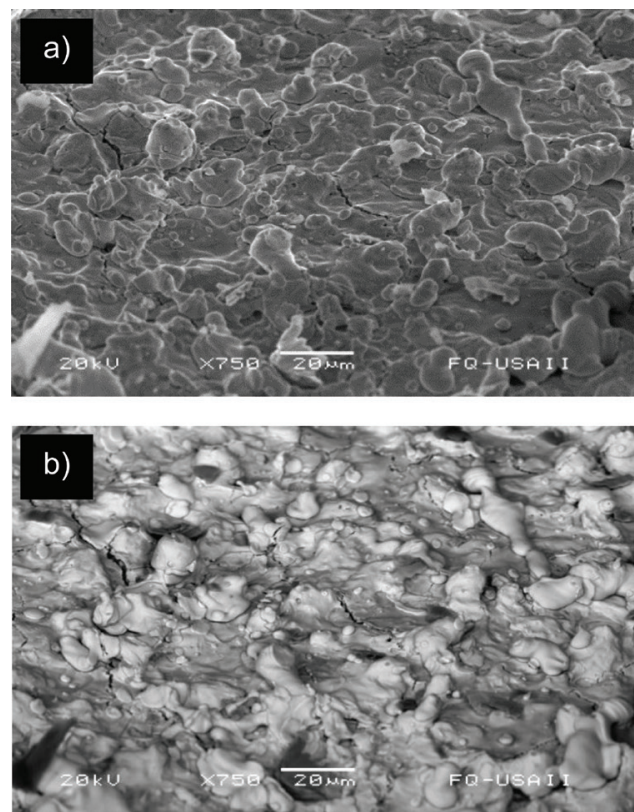

Figure 9: a) SEI of the top surface Tungsten electrode, b) Backscattered electron image (BEI) from the same region that (a), The W cover uniformly al the copper electrode surface. Copper electrode surface shows the formation of drops.

The EDX elemental mapping shown in the Fig. 10 and Fig. 11 shows that the copper concentration over the copper electrode increases from the central region towards the edge of copper electrode. This apparent enrichment is due to the thinning of the Tungsten films; the film is thicker at the central region and become thinnest near the copper electrode edge.
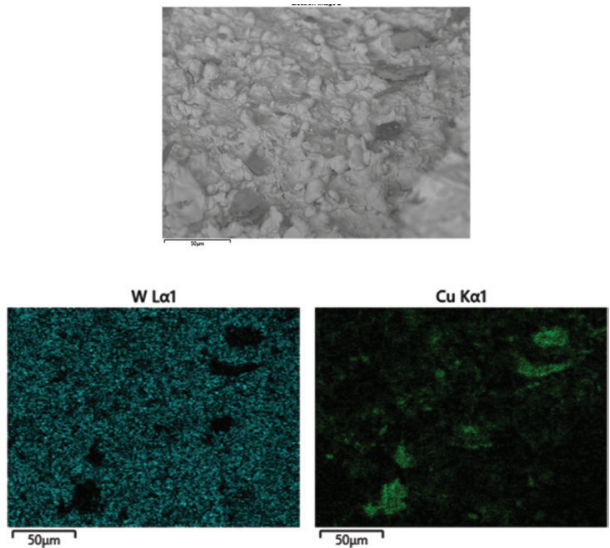

Figure 10: Surface located between center and the border of the copper electrode, where the film formed by Tungsten is thicker and almost uniform. Mapping of $\mathrm{W}$ and $\mathrm{Cu}$ shows regions with copper concentrations. 


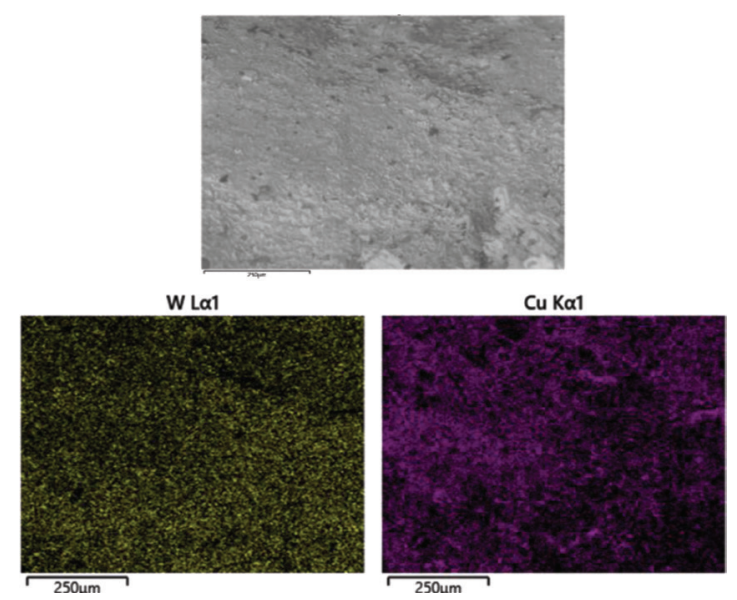

Figure 11: Mapping of $\mathrm{W}$ and $\mathrm{Cu}$ near to the border of copper electrode, the W film is thinner and can be observed an upper and more homogeneous copper concentration compared with the elemental distribution found toward the center of copper electrode.

\section{Conclusions}

The purpose of the work was to analyze the damage caused to the both needle Tungsten and the copper electrode by the hot plasma discharge. For this, we carry out a series of tests and analyzes with which we conclude that the effects of the plasma discharges are greater in the Tungsten needle, due to the small size spot of the plasma discharge produced by the FN-II small plasma focus device and to the direct impact it has on Tungsten needle.

The impact of the plasma column caused its erosion after approximately 70 shots (discharges), distributing much of the material on the copper electrode, presenting a morphological change.

When analyzing the micro-hardness in a previous work, where there was no needle, a marked change was shown in two areas of the electrode. In this case the changes are minor but more distributed in the electrode, marking an area of greater hardness at greater depth of the electrode, as a result of the immediate heating and also the immediate cooling suffered by the discharge electrode to discharge. It also has a greater hardness in the center of the electrode, a result of the rapid cooling heating that occurs as mentioned.

\section{Acknowledgements}

The authors are thankful to J. Herrera, J. Rangel, H. H. Hinojosa, I. Puente, I. Reyes, F. Castillo, O. Flores and E. Vazquez-Velez for their technical assistance. DGAPA IN102916 and CONACyT228644 sponsored this research.

\section{References}

[1] F. Castillo et al., Braz. J. of Phy. 32, 3 (2002). https://doi.org/10.1590/S0103-97332002000100002

[2] F. Castillo et al., Rad. Prot. Dos. 101, 557 (2002). https://doi.org/10.1093/oxfordjournals.rpd.a006048

[3] F. Castillo et al., Plas. Phy. Cont. Fus. 45, 289 (2003). https://doi.org/10.1088/0741-3335/45/3/309

[4] M. Zakaullah, K. Alamgir, G. Murtaza and A. Waheed, Plas. Sou. Sci. Technol. 9, 592 (2000). https://doi.org/10.1088/0963-0252/9/4/315

[5] J. Pouzo, H. Acuña, M. Milanese, and R. Moroso, Eur. Phys. J. D 21, 97 (2002). https://doi.org/10.1140/epjd/e2002-00183-2

[6] F. Castillo, I. Gamboa-deBuen, J.J.E. Herrera, J. Rangel and S. Villalobos, App. Phy. Lett. 92, 051502 (2008). https://doi.org/10.1063/1.2840191

[7] F. Castillo, I. Gamboa-deBuen, J.J.E. Herrera, J. Rangel; $29^{\text {th }}$ ICPIG 2009 16-19 (2009).

[8] R. Reed-Hill and R. Abbaschian, Physical Metallurgy Principles (Boston, PWS-Kent Pub.1992) $3^{\text {rd }}$ Edition, Chapter 20, 688-691.

[9] F. Castillo, J.J.E. Herrera and J. Rangel, AIP Conf. Proc. Series 875, 405 (2006). https://doi.org/10.1063/1.2405975

[10] F. Di Lorenzo et al., J. Appl. Phys. 102, 033304, (2007). https://doi.org/10.1063/1.2767829

[11] C. Moreno et al., Appl. Phys. Letts. 89, 091502 (2006). https://doi.org/10.1063/1.2335631

[12] S. Hussain et al., Plasma Sources Sci. Technol. 14, 61 (2005). https://doi.org/10.1088/0963-0252/14/1/008

[13] J. García Molleja et al., Surf. Coat. Tech. 218, 142 (2013). https://doi.org/10.1016/j.surfcoat.2012.12.043

[14] J. García Molleja et al., Surf. Interface Anal. 47, 728 (2015). https://doi.org/10.1002/sia.5770

[15] V.N. Pimenov et al., Nukleonika 53, 111 (2008).

[16] R. L. Klueh et al., J. Nucl. Mater. 455, 307 (2002). https://doi.org/10.1016/S0022-3115 (02)01082-6

[17] G. Sánchez, G. Grigioni and J. Feugeas, Surf. Coat. Technol. 70, 181 (1995). https://doi.org/10.1016/0257-8972(94)02265-R

[18] Michael F. Ashby, Materials Selection in Mechanical Design, Pergamum Press, Third Edition (2005). 
旬

CHITKARA
Journal of Nuclear Physics, Material Sciences, Radiation and Applications

Chitkara University, Saraswati Kendra, SCO 160-161, Sector 9-C, Chandigarh, 160009, India

Volume 7, Issue 2

February 2020

ISSN 2321-8649

Copyright: [C 2020 R. S. Monzamodeth and B. Campillo] This is an Open Access article published in Journal of Nuclear Physics, Material Sciences, Radiation and Applications (J. Nucl. Phy. Mat. Sci. Rad. A.) by Chitkara University Publications. It is published with a Creative Commons Attribution- CC-BY 4.0 International License. This license permits unrestricted use, distribution, and reproduction in any medium, provided the original author and source are credited. 